\title{
Synthesis, characterization and electrospinning of poly(vinyl caprolactam-co-hydroxymethyl acrylamide) to create stimuli-responsive nanofibers
}

Edurne González*, Margaret W. Frey

Department of Fiber Science and Apparel Design, Cornell University, Ithaca, NY 14853, USA (edurne_gonzalez001@ehu.eus)

\section{Keywords}

Stimuli-responsive nanofibers, thermo-responsive nanofibers, poly(vinyl caprolactam) PVCL, poly(hydroxymethyl acrylamide) PNMA, electrospinning

\begin{abstract}
Poly(vinyl caprolactam) (PVCL) is an especially attractive temperature-responsive polymer due to its biocompatibility and the fact that its lower critical solution temperature (LCST) is in the physiological range $\left(32-34^{\circ} \mathrm{C}\right)$. Here, PVCL was copolymerized with hydroxymethyl acrylamide (NMA) and electrospun to create PVCL based temperature-responsive chemical hydrogel nanofibers for the first time. Field emission scanning electron microscopy (FESEM) was used to study fiber morphology. The thermal curing process of the nanofibers was analyzed by attenuated total reflectance-fourier transform infrared spectroscopy (ATR-FTIR), differential scanning calorimetry (DSC) and thermogravimetric analysis (TGA). The created "smart" hydrogel nanofibers responded quickly and reversibly to changes in temperature and showed a temperature controlled rhodamine B dye release. The unique properties offered by these novel materials show promise for applications in biosensors, controlled drug delivery and microfluidic systems.
\end{abstract}

\section{Introduction}

"Smart" or stimuli-responsive polymers respond to small changes in their environment, such as $\mathrm{pH}$, temperature, ionic strength, etc., by dramatically changing their chemical and physical properties. These materials have gained extraordinary relevance over the last decades due to their promising applications in integrated microfluidic systems, biosensors, bioengineering, drug delivery, diagnostics, tissue engineering, coatings and textiles [1-4].

Temperature-responsive polymers, as one kind of "smart" polymers, respond to changes in the temperature and undergo a phase transition at the lower critical solution temperature (LCST). At temperatures below the LCST these macromolecules are hydrophilic, while at temperatures above LCST they become hydrophobic and collapse [4]. Among the different temperatureresponsive polymers, poly(N-isopropylacrylamide) (PNIPAm) and poly(vinyl caprolactam) 
(PVCL) are especially attractive because their LCST is in the physiological range $\left(32-34{ }^{\circ} \mathrm{C}\right)$. Although PNIPAm has been more deeply studied in the last years, PVCL is a better alternative for biological applications due to its higher biocompatibility $[5,6]$.

Crosslinked temperature-responsive polymers form hydrogels that exhibit dramatic swelling or shrinking below and above the LCST. Hydrogels can be physically or chemically crosslinked. Physical hydrogels are held together by hydrophobic non-covalent bonds, whereas chemical hydrogels are obtained by chemical crosslinking [3]. Nowadays, the most significant weakness of stimuli-responsive hydrogels is their slow response. Thus, fast-acting hydrogels are necessary [4]. Since swelling kinetics of hydrogels are inversely proportional to the square of their dimension, scientist have recently shown that changing the morphology of the hydrogels from films to nanofibers significantly enhances the rate of volume change [7-10]. Therefore, the creation of thermo-responsive nanofibers is a new research area of increasing interest.

Currently, electrospinning is the most versatile technique to create polymeric nanofiber nonwoven mats [11-14]. Most of the works related to the electrospinning of PNIPAm have been published in the last eight years. Researches have electrospun PNIPAm polymer with the objective to obtain thermo-responsive nanofibers with reversible wettability [15-17], swellingshrinking capacity [7, 18, 19], "on-off" luminescence [20-22] or controlled drug release [23-25]. Interestingly, there are few works in the literature related to the electrospinning of PVCL homopolymers and copolymers [26-30]. Webster and co-workers [30] electrospun PVCL homopolymer for the first time. They synthesized PVCL homopolymers with different molecular weights and chain end functional groups and studied effect of the electrospinning parameters on the morphology of the final nanofibers. They also electrospun mixtures of PVCL/cellulose acetate to obtain composite nanofibers with reversible wettability. Paaver et al.[28] studied the spinnability of the synthetic graft copolymer "Soluplus" (poly(vinyl caprolactam-vinyl acetate-ethylene glycol) graft copolymer) and the ability of the nanofibers to act as drug delivery systems. Yu et al. [26] synthesized and electrospun poly(vinyl caprolactamco-methyl methacrylate) (P(VCL-co-MMA)) copolymers and studied the wettability, swelling capacity and drug release behavior of the created physical hydrogel nanofiber mats. In addition, Pich et al. [29] electrospun poly(caprolactone) (PCL) together with PVCL nanogels to obtain composite nanofibers in a single electrospinning process. The addition of the PVCL nanogels led to hydrophobic PCL nanofibers with controlled swelling and regulated degradability. Later, they were also able to load the nanogels with $\mathrm{ZnO}$ obtaining microfibers capable of releasing zinc ion under physiological conditions [27].

Here we aim to create, for the first time, PVCL chemical hydrogel nanofibers with reversible swelling capacity. Since crosslinked polymers are not soluble in any solvent, their 
electrospinning is not possible. Therefore, when creating chemical hydrogel nanofibers, the chemical crosslinking of the nanofiber could occur only after the electrospinning process. As a consequence, in this work, we copolymerized PVCL with hydroxymethyl acrylamide (NMA), which is a heat induced self-crosslinkable monomer. NMA has been previously copolymerized with PNIPAm to create PNIPAm based chemical hydrogels [18, 21, 31, 32], however, this is the first time that poly(vinyl caprolactam-co-hydroxymethyl acrylamide) (P(VCL-co-NMA)) polymers are electrospun. We synthesized P(VCL-co-NMA) polymers of different composition and found the appropriate electrospinning parameters to obtain uniform bead-free nanofibers. The crosslinking process of the nanofibers was studied by attenuated total reflectance-fourier transform infrared (ATR-FTIR), differential scanning calorimetry (DSC) and thermogravimetric analysis (TGA). In addition, we analyzed the effect of the polymer composition on the morphology and swelling-shrinking capacity of the nanofiber mats. Finally, we also studied the temperature dependent controlled dye release capacity of the nanofiber mats.

\section{Experimental work}

\subsection{Materials}

Vinyl caprolactam (VCL), 2,2'-azobis (2-methylpropionitrile) (AIBN) and dimethylformamide (DMF), Methanol-d $\mathrm{d}_{4}\left(\mathrm{CD}_{3} \mathrm{OD}\right)$ were all purchased form Sigma-Aldirch. Hydroxymethyl acrylamide (NMA) was purchased from Santa Cruz Biotechnology Inc. All chemicals were used as received without further purification. Ultrapure water (Mili-Q, Millipore) was used in all the experiments.

\subsection{Synthesis of $P(V C L-c o-N M A)$ polymers of different composition}

All polymers were synthesized by free radical solution polymerization using DMF as solvent. Reactions were performed in a three neck round bottom flask equipped with a reflux condenser and a $\mathrm{N}_{2}$ inlet. First, the monomers and the DMF were added to the flask, the temperature was increased to $60{ }^{\circ} \mathrm{C}$ and the reaction mixture was stir for 15 minutes under $\mathrm{N}_{2}$ flow. Then, the initiator (AIBN) was added, dissolved in $5 \mathrm{~mL}$ of the DMF, to start the reaction. All the reactions were performed at $60{ }^{\circ} \mathrm{C}$ for 16 hours under continuous $\mathrm{N}_{2}$ flow. The initial monomer concentration was $1.6 \mathrm{mmol} / \mathrm{mL}$ and the initiator concentration was $0.5 \mathrm{~mol} \%$ respect to the monomers in all the cases. Different polymerizations were performed varying the monomer mole ratios (see Table 1). Polymerizations were stopped by cooling the reaction mixture to room temperature and precipitating the polymer in diethyl ether (by adding the solution dropwise). The polymer was isolated by vacuum filtration and drying at $40{ }^{\circ} \mathrm{C}$ under vacuum overnight. Synthesized polymers were dialyzed against ultrapure water in order to remove the residual monomers and solvent. Spectra/Por (MwCO: 12,000-14,000) was used as membrane. The entire dialysate volume was changed for fresh dialysate solution at least once a day. 
Dialysis was allowed to run until the conductivity of the dialysate was close to that of the ultrapure water $(2 \mu \mathrm{S} / \mathrm{cm})$. Finally, polymers were recovered by freeze-drying.

Table 1. Polymerization recipes used to synthesize $\mathrm{P}$ (VCL-co-NMA) polymers by solution polymerization. The amount of AIBN was $0.5 \mathrm{~mol} \%$ respect to the monomers and the monomer concentration was $1.16 \mathrm{mmol} / \mathrm{mL}$ in all the cases.

\begin{tabular}{|c|c|c|}
\hline Reaction Name & VCL $(\mathrm{mol} \%)$ & NMA (mol \%) \\
\hline PN1 & 95 & 5 \\
\hline PN2 & 90 & 10 \\
\hline PN3 & 80 & 20 \\
\hline
\end{tabular}

\subsection{Characterization of $P(V C L-c o-N M A)$}

\subsubsection{Nuclear magnetic resonance (NMR)}

${ }^{1} \mathrm{H}-\mathrm{NMR}$ experiments were recorded at room temperature with an INOVA 600 spectrometer operating at $600 \mathrm{MHz}$ and using $\mathrm{CD}_{3} \mathrm{OD}$ as solvents.

\subsubsection{Gel permeation chromatography $(G P C)$}

A Waters ambient-temperature GPC equipped with a Waters 1515 isocratic HPLC pump and a Waters 2414 refractive index detector at $50{ }^{\circ} \mathrm{C}$. Dimethyl sulfoxide (DMSO) with $0.05 \mathrm{M}$ of lithium bromide was used as mobile phase with a flow rate of $0.5 \mathrm{~mL} / \mathrm{min}$. Obtained molecular weight values were referred to polystyrene standards.

\subsubsection{Lowest critical solution temperature (LCST)}

LCST of the polymers was determined by measuring the transmittance at $500 \mathrm{~nm}$ (Spectramax 384 spectrophotometer) of a polymer solution in ultrapure water (1 wt \%) at different temperatures. The LCST of the copolymer was defined as the temperature at which transmittance of the solution decreased in $50 \%$.

\subsection{Electrospinning and curing of fiber mats}

The electrospinning apparatus used in this work consisted of syringe ( $1 \mathrm{~mL}$ plastic (DB)) equipped with a needle (25 G (Small Parts Inc.)), a syringe pump (Harvard Apparatus), a high voltage power supply (Gamma High Voltage Research Inc.) and a grounded collector (a copper substrate covered by aluminum foil). Nanofibers were crosslinked by placing them into a preheated oven. Different curing temperature (120, 150180 and $210{ }^{\circ} \mathrm{C}$ ) and times (from 30 minutes up to 15 hours) were investigated. 


\subsection{Characterization of nanofiber mats}

\subsubsection{Fiber morphology by field emission scanning electron microscopy (FESEM)}

FESEM imaging was carried out on a LEO 1550 FESEM to analyze fiber morphology. Samples were left uncoated and images were obtained using an acceleration voltage of $1.5 \mathrm{kV}$ at a working distance of $5 \mathrm{~mm}$. Image ${ }^{\mathrm{TM}}$ open source software (National Institutes of Health) was used to measure the mean average fiber diameters. Fifty measurements were taken for each sample from three separate images.

\subsubsection{Attenuated total reflectance-fourier transform infrared (ATR-FTIR) spectroscopy}

Curing process of the polymer nanofiber mats was analyzed by ATR-FTIR spectrophotometer (Nicolet Magna-IR 560) before and after crosslinking. Spectra were taken in the range of 4000$600 \mathrm{~cm}^{-1}$ wavenumbers. Each scan was an average of 200 scans obtained at a resolution of $4 \mathrm{~cm}^{-}$ 1 .

\subsubsection{Differential scanning calorimetry (DSC)}

Nanofiber mats were analyzed by differential scanning calorimetry (DSC, Q2000, TA Instruments). Samples were heated from 0 to $300{ }^{\circ} \mathrm{C}$ at $10{ }^{\circ} \mathrm{C} / \mathrm{min}$, cooled to $0{ }^{\circ} \mathrm{C}$ at $10{ }^{\circ} \mathrm{C} / \mathrm{min}$ and re-heated to $300{ }^{\circ} \mathrm{C}$ at $10{ }^{\circ} \mathrm{C} / \mathrm{min}$.

\subsubsection{Thermogravimetric analysis (TGA)}

Thermogravimetric analysis (TGA 500, TA instrument) was used to analyze the thermal properties of the nanofiber mats. All TGA analyses were performed between 30 and $800{ }^{\circ} \mathrm{C}$ and at a ramp rate of $10^{\circ} \mathrm{C} / \mathrm{min}$ under a nitrogen atmosphere by maintaining a flow rate of 60 $\mathrm{mL} / \mathrm{min}$ for the sample purge and $40 \mathrm{~mL} / \mathrm{min}$ for the balance purge.

\subsubsection{Thermo-responsive behavior of nanofibers}

To determine thermo-responsive behavior of the nanofibers, their swelling-shrinking ability in water below and above the LCST temperature was determined. First, small pieces (around $3 \times 2$ $\mathrm{cm}$ and $\sim 10 \mathrm{mg}$ ) of crosslinked nanofiber mat were place in ultrapure water at $52 \pm 2{ }^{\circ} \mathrm{C}$ for 5 minutes. Then, the nanofiber mat was collected and weighed. Subsequently, the nanofibers was immersed in ultrapure water at $23 \pm 1{ }^{\circ} \mathrm{C}$ for another 5 minutes and afterwards collected and weighed. This process was repeated four times and the weight of the swollen or shrunk nanofiber mat at each temperature was measured. The swelling ratio at each temperature was calculated using the following equation [32]:

$$
\text { Swelling ratio }(\%)=\left(\left(w_{\text {swell }}-w_{\text {dry }}\right) / w_{\text {dry }}\right) \times 100
$$


where $w_{\text {swell }}$ and $w_{d r y}$ are the weights of the swollen and dry nanofibers respectively.

\subsection{Controlled dye release of nanofibers.}

For dye release experiment, $1 \mathrm{wt} \%$ of rhodamine B (respect to the polymer) was incorporated into PN2 nanofiber mat by dissolving the dye directly in the initial polymer solution (electrospinning dope) and electrospinning it. In this case, the created pink nanofiber mat (pictures can be found in the supplementary material (SM)) was cured at $180^{\circ} \mathrm{C}$ for just 30 minutes to avoid the degradation of rhodamine B. Later, $3 \times 2 \mathrm{~cm}$ pieces were cut $(\sim 10 \mathrm{mg})$ and immersed in $15 \mathrm{~mL}$ of DI water at two different temperatures; $23 \pm 2$ and $52 \pm 2{ }^{\circ} \mathrm{C}$. Rhodamine $\mathrm{B}$ release was calculated by measuring the absorbance of the water solution (a small aliquot was withdrawn and then put back in the solution) at $550 \mathrm{~nm}$ by UV-visible spectroscopy (Perkin Elmer).

\section{Result and discussion}

\subsection{Synthesis and characterization of P(VCL-co-NMA)}

Table 2 summarizes the main properties of the synthesized P(VCL-co-NMA) polymers in terms of molecular weight $(\mathrm{Mw})$, molar mass dispersity values $(Đ)$, molar composition and LCST.

Table 2. Characterization of polymers synthesized by free radical solution polymerization (Table 1)

\begin{tabular}{|c|c|c|c|c|c|}
\hline & & & \multicolumn{2}{|c|}{ Real composition $(\mathrm{mol} \%)^{b}$} & \\
\hline Name & $\mathrm{Mw}(\mathrm{g} / \mathrm{mol})^{\mathrm{a}}$ & $\bigoplus^{\mathrm{a}}$ & VCL & NMA & $\operatorname{LCST}\left({ }^{\mathrm{D} C}\right)$ \\
\hline PN1 & 25400 & 1.5 & 92 & 8 & 31.5 \\
\hline PN2 & 28400 & 1.8 & 65 & 35 & 33.7 \\
\hline PN3 & 28800 & 1.7 & 56 & 44 & 37.4 \\
\hline
\end{tabular}

${ }^{\mathrm{a}}$ Determined by GPC, ${ }^{\text {b }}$ determined by NMR

The molar composition of the copolymers was determined by ${ }^{1} \mathrm{H}-\mathrm{NMR}$ spectroscopy integrating the peaks corresponding to VCL protons at $2.4 \mathrm{ppm}$ and VCL+ NMA protons at $4.5 \mathrm{ppm}$ (see Figure 1) and using equation 5:

$$
\begin{gathered}
\mathrm{S}_{\mathrm{VCL}}=2 \mathrm{H} \\
\mathrm{H}=\mathrm{S}_{\mathrm{VCL} / 2} \\
\mathrm{~S}_{\mathrm{VCL}+\mathrm{NMA}}=\mathrm{H}_{\mathrm{VCL}}+\mathrm{x} 2 \mathrm{H}_{\mathrm{NMA}} \\
\mathrm{S}_{\mathrm{VCL}+\mathrm{NMA}}=\left(\mathrm{S}_{\mathrm{VCL} / 2}\right)+\mathrm{x} 2\left(\mathrm{~S}_{\mathrm{VCL} / 2}\right) \\
\mathrm{x}=\frac{\mathrm{S}_{\mathrm{VCL}+\mathrm{NMA}}-\left(\mathrm{S}_{\mathrm{VCL} / 2}\right)}{2\left(\mathrm{~S}_{\mathrm{VLL} / 2}\right)}
\end{gathered}
$$


Where $\mathrm{S}_{\mathrm{VCL}}$ is the signal corresponding to VCL protons at $2.4 \mathrm{ppm}, \mathrm{S}_{\mathrm{VCL}+\mathrm{NMA}}$ is the signal corresponding VCL+ NMA protons at $4.5 \mathrm{ppm}$ and $x$ is the VCL/NMA mole ratio,

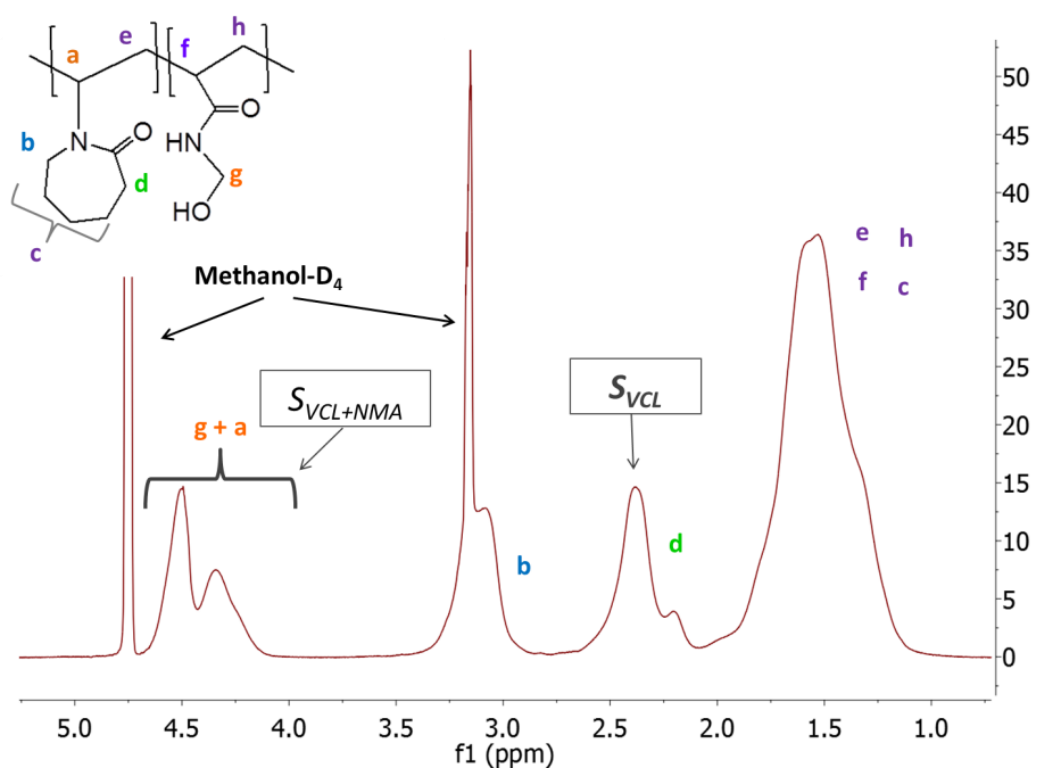

Figure 1. ${ }^{1} \mathrm{H}-\mathrm{NMR}$ spectra of PN2 copolymer

For comparison purposes, quantitative ${ }^{13} \mathrm{C}-\mathrm{NMR}$ experiment of PN2 polymer was also

performed obtaining very similar results in the composition of the polymer. Result can be found in the SM.

In all the cases, the NMA mol \% of the final copolymers was larger than in the initial feed. The reason is attributed to the low reactivity ratio of VCL monomer, as found by other authors [33, 34].

LCST of the copolymers was estimated from the variation in the transmittance of a $1 \mathrm{wt} \%$ water solution with temperature. Results are shown in Figure 2. For the sake of comparison, the result for PVCL homopolymer is also shown. As expected, the incorporation of NMA hydrophilic monomer, made stretching of polymer chains at elevated temperatures more difficult, increasing the LCST of the copolymer. The higher the NMA content in the copolymer, the higher the LCST. The same trend was observed by other authors when NMA was copolymerized to PNIPAm polymers [21, 31, 32]. 


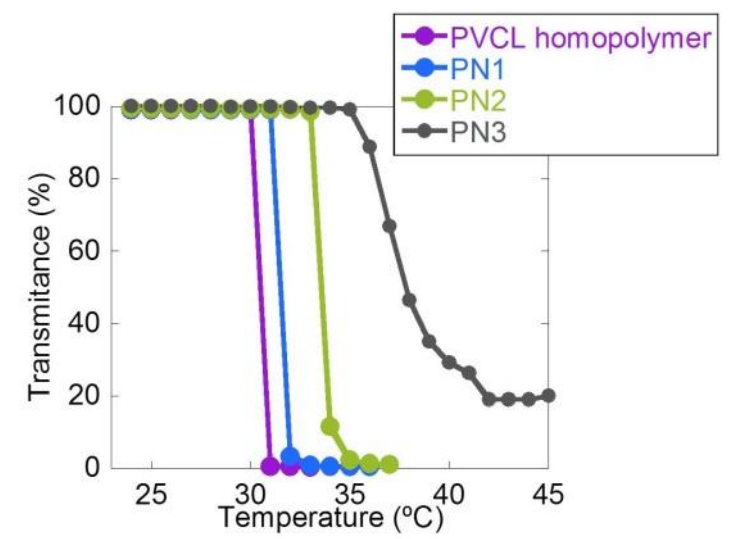

Figure 2. Variation in the transmittance at $500 \mathrm{~nm}$ versus temperature of $1 \mathrm{wt} \%$ water solutions of each polymer.

\subsection{Electrospinning of P(VCL-co-NMA) polymers of different compositions}

Our first objective was to find the best processing parameter to obtain continuous bead-free nanofibers. To this end, water solutions of PN2 copolymer were electrospun at different conditions changing the solution concentration, the flow rate, the voltage and the tip to collector distance. Obtained results are summarized in Table 3.

Table 3. Process parameters used to electrospun PN2 copolymer. Ultrapure water was used as solvent in all the cases (FESEM images can be found in SM)

\begin{tabular}{|c|c|c|c|c|}
\hline $\begin{array}{c}\text { Conc. } \\
(\mathbf{w} / \mathbf{v} \%)\end{array}$ & $\begin{array}{c}\text { Flow rate } \\
(\boldsymbol{\mu} \mathbf{L} / \mathbf{m i n})\end{array}$ & $\begin{array}{c}\text { Voltage } \\
(\mathbf{k V})\end{array}$ & $\begin{array}{c}\text { Tip-collector } \\
\text { distance }(\mathbf{c m})\end{array}$ & Result \\
\hline 50 & $\begin{array}{c}\text { Varied from } \\
2.5 \text { to } 10\end{array}$ & $\begin{array}{c}\text { Varied } \\
\text { from } 10 \\
\text { to } 15\end{array}$ & 10 & $\begin{array}{c}\text { Fibers with beads in all the } \\
\text { cases and solvent droplets in } \\
\text { the collected fiber mat }\end{array}$ \\
\cline { 4 - 5 } & 2.5 & 13 & 15 & $\begin{array}{c}\text { Fibers with beads in all the } \\
\text { cases }\end{array}$ \\
\cline { 4 - 5 } & & 15 & $\begin{array}{c}\text { Continuous bead-free fibers but } \\
\text { difficult to peel-off from the } \\
\text { substrate }\end{array}$ \\
\cline { 3 - 5 } & & & 23 & $\begin{array}{c}\text { Continuous bead-free fibers. } \\
\text { Easy to peel-off from the } \\
\text { substrate }\end{array}$ \\
\hline
\end{tabular}

When a $50 \mathrm{wt} \%$ solution was electrospun, continuous fibers with beads were obtained for all the different flow rates and voltages. Furthermore, solvent droplets were observed in the collected fiber mat when the tip to collector distance was $10 \mathrm{~cm}$. As a consequence, solution concentration was increased in order to avoid the bead formation and the tip-to collector distance was increase to give enough time to the solvent (water) to evaporate before the fibers reached the collector $[35,36]$. As a result, we came to the conclusion that the most appropriate processing parameters to obtain a continuous bead-free nanofiber mats that could be easily peeled-off from the substrate were the following ones: a concentration of $55 \mathrm{wt} \%$ in ultrapure water, a flow rate of $2.5 \mu \mathrm{L} / \mathrm{min}$, a voltage of $13 \mathrm{kV}$ and a tip to collector distance of $23 \mathrm{~cm}$. 
These parameters were used to electrospun the synthesized three different copolymers. The obtained fibers are shown in Figure 3.

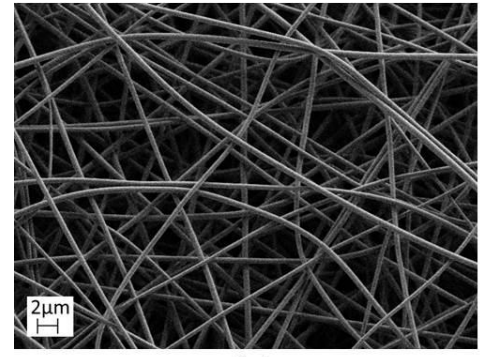

(a)

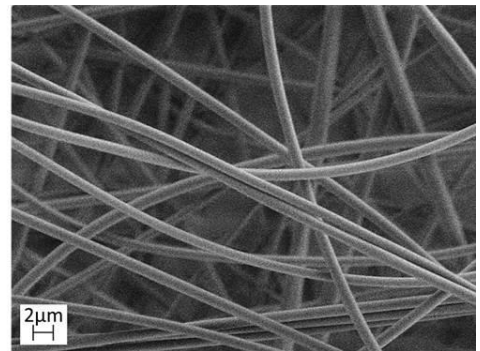

(b)

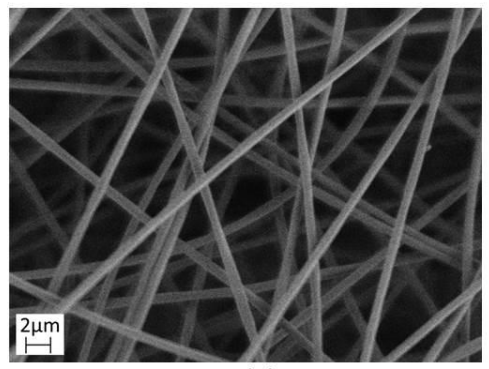

(c)

Figure 3. FESEM micrograph of nanofibers obtained by electrospinning $55 \mathrm{wt} \%$ solutions in DI water of polymers with different compositions: (a) PN1, (b) PN2 and (c) PN3. All experiments were performed at $13 \mathrm{kV}$ using a flow rate of $2.5 \mu \mathrm{L} / \mathrm{min}$ and a tip to collector distance of $23 \mathrm{~cm}$.

Figure 4 compares the average diameter of the obtained nanofibers as the NMA mol $\%$ in the copolymer increases. No clear trend was observed.

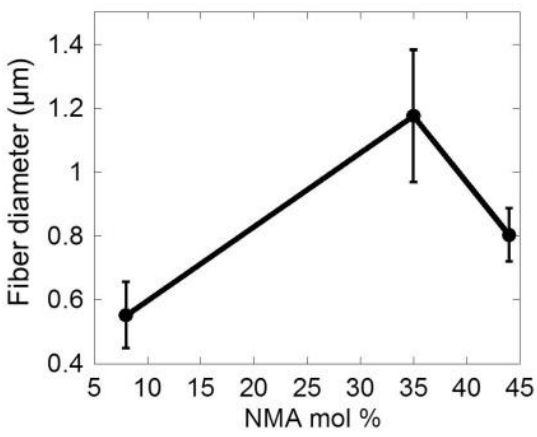

Figure 4. Evolution of the average fiber diameter as the NMA content in the copolymer increases (Fiber in Figure 3).

\subsection{Curing of P(VCL-co-NMA) nanofiber mat}

As it has been previously reported in the literature, NMA monomer can self-crosslink by the use of heat $[18,31,32]$. Figure 5 shows the crosslinking mechanism for P(VCL-co-NMA) copolymers. 


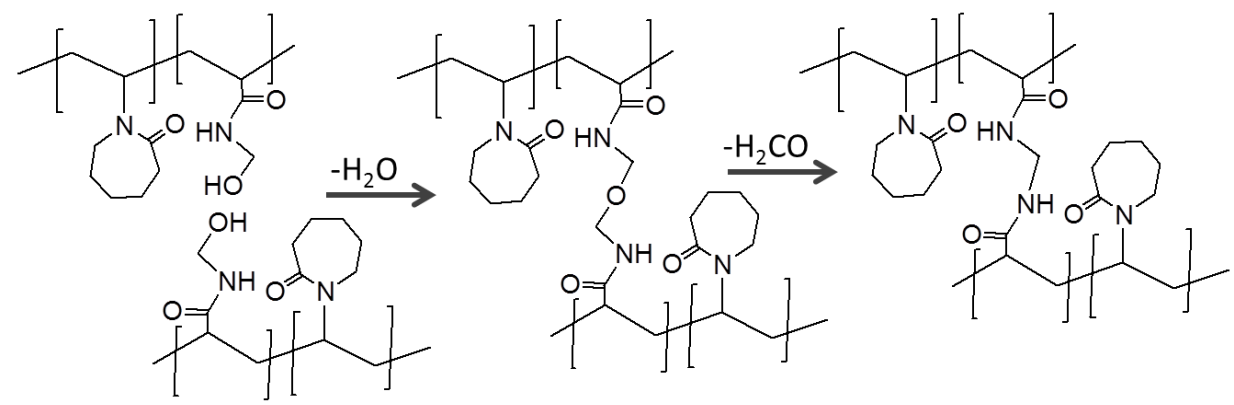

Figure 5. P(VCL-co-NMA) polymer crosslinking reaction

In order to find the appropriate curing conditions, a nanofiber mat spun from PN3 polymer (44 mol $\%$ of NMA) was cured at different temperatures $\left(120,150,180\right.$ and $\left.210{ }^{\circ} \mathrm{C}\right)$ for different periods of time (from 30 minutes up to 15 hours) and samples were analyzed by FT-IR spectrometry. As an example, Figure 6 shows the spectra of the sample cured at $180{ }^{\circ} \mathrm{C}$ for different times. As the curing reaction occurred, the intensity of N-H and O-H stretching (3300 $\left.\mathrm{cm}^{-1}\right)$ and $\mathrm{C}-\mathrm{O}$ stretching $\left(1100 \mathrm{~cm}^{-1}\right)$ bands decreased, while the rest of the peaks were maintained equal. In order to compare the effectiveness and crosslinking rate of different temperatures, the ratio between $\mathrm{C}-\mathrm{O}$ stretch and $\mathrm{C}=\mathrm{O}$ stretch $(1700 \mathrm{~cm}-1)$ was plotted against the curing time for each temperature as it shown in Figure 7.

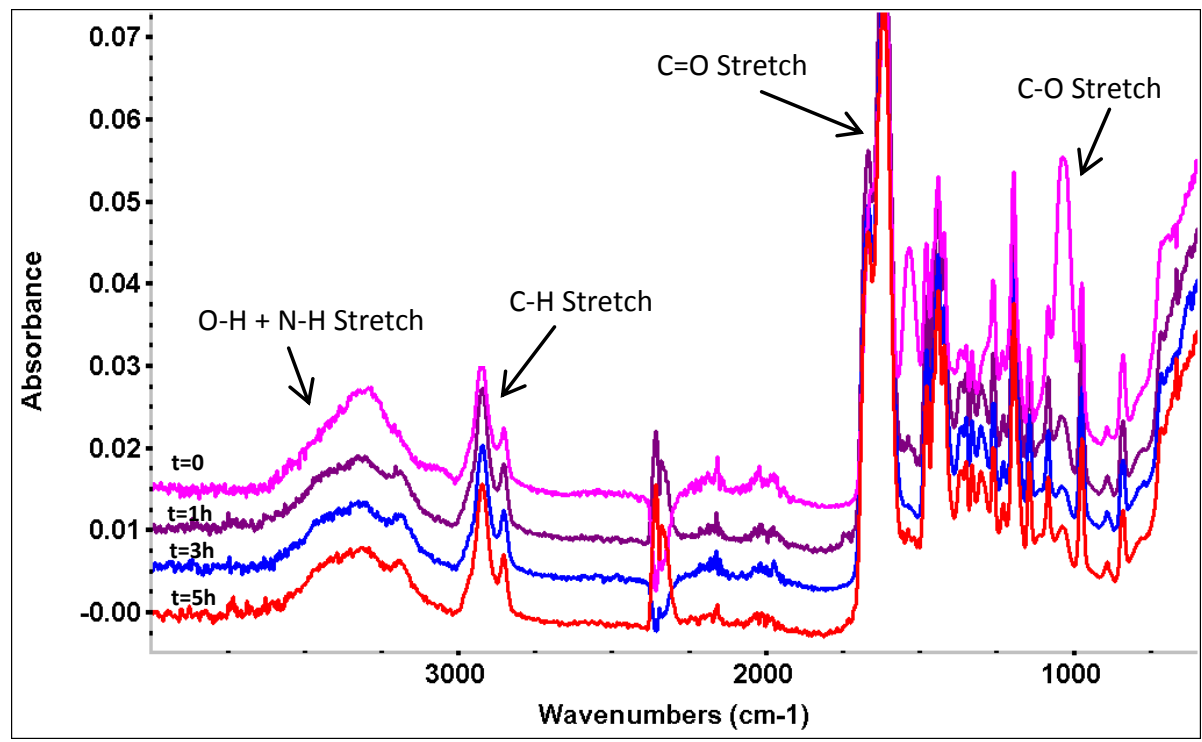

Figure 6. FTIR spectra of PN3 nanofibers cured at $180{ }^{\circ} \mathrm{C}$ for different times 


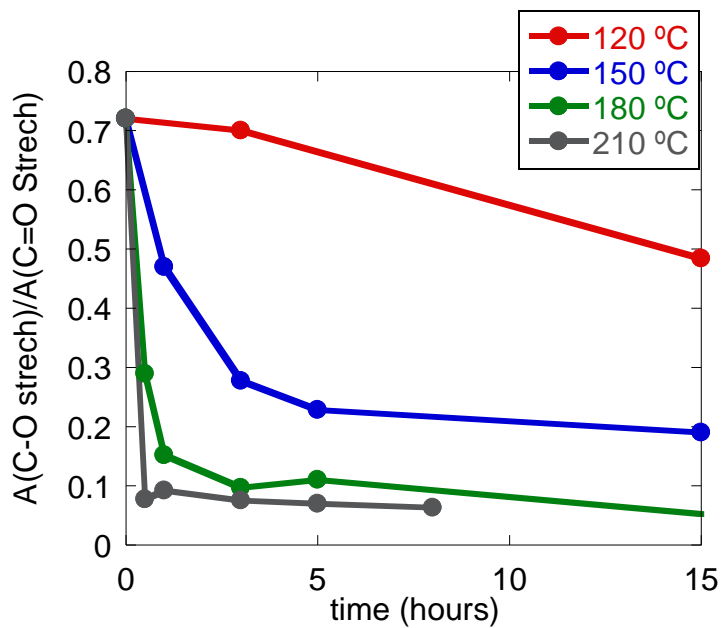

Figure 7. Ratio between $\mathrm{C}-\mathrm{O}$ stretch $\left(1100 \mathrm{~cm}^{-1}\right)$ and $\mathrm{C}=\mathrm{O}$ stretch $\left(1700 \mathrm{~cm}^{-1}\right)$ in front of curing time for PN3 nanofibers.

As expected, crosslinking rate increased with temperature. Crosslinking rate was very slow at $120^{\circ} \mathrm{C}$. After $15 \mathrm{~h}$ of curing at $120^{\circ} \mathrm{C}$ the crosslinking ratio was similar to that observed after $1 \mathrm{~h}$ at $150{ }^{\circ} \mathrm{C}$ (Figure 7). The most effective temperatures were found to be 180 and $210{ }^{\circ} \mathrm{C}$. Complete curing was observed after 30 minutes at $210^{\circ} \mathrm{C}$. Intriguingly, lower temperatures (from 80 to $110^{\circ} \mathrm{C}$ ) have been used in other works to crosslink P(NIPAm-co-NMA) nanofibers $[18,21,31,32]$. The reason might be attributed to the higher glass transition temperature $(\mathrm{Tg})$ of PVCL compared to PNIPAm; $194{ }^{\circ} \mathrm{C}$ for PVCL (measured experimentally) and $\sim 130{ }^{\circ} \mathrm{C}$ for PNIPAm [37]. Crosslinking reaction only occurs when NMA moieties come into contact in the presence of heat. As polymer chains start to move at temperatures above their $\mathrm{Tg}$, the contact between NMA moieties is increased at these temperatures, enhancing the crosslinking reaction. Thus, the higher temperatures are necessary to crosslink polymers of higher Tg.

Figure 8 (a) shows the TGA thermograph of the as spun PN3 nanofiber mat (containing 44 mol $\%$ of NMA) as well as the mats cured at $210^{\circ} \mathrm{C}$ for 1 and 7 hours. In the case of the as spun sample (not cured), an initial decrease in the weight was observed at around $180^{\circ} \mathrm{C}$. This first transition was not observed in the cured samples, so, it was associated to the water and formaldehyde release caused by the crosslinking reaction (see Figure 5). The second transition (starting at $290^{\circ} \mathrm{C}$ ) was associated with the polymer degradation. Thermographs corresponding to the samples cured for 1 and 7 hours overlap each other demonstrating that there was no degradation of polymer after 7 hours of curing at $210^{\circ} \mathrm{C}$. In addition, Figure 8 (b) compares the TGA thermograph of the as spun fibers of the three different copolymers containing different NMA mol \%. The amount of reactive sites in the sample increases as the amount of NMA molecules increases, leading to a larger decrease in weight as more water and formaldehyde molecules are lost. 


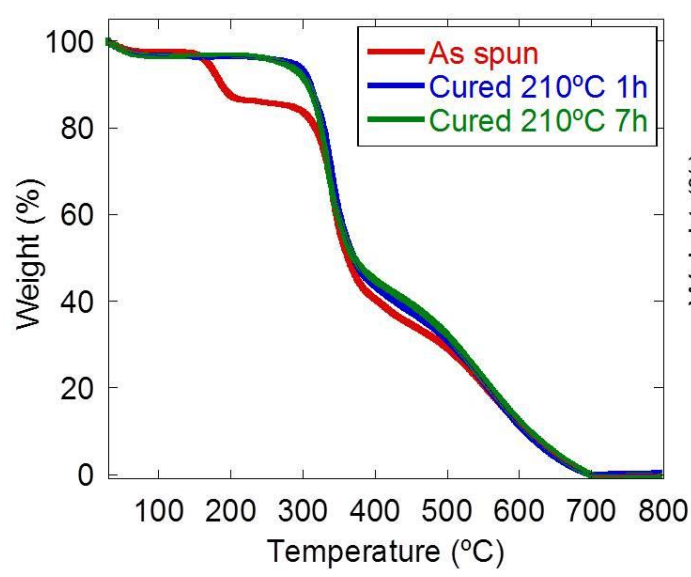

(a)

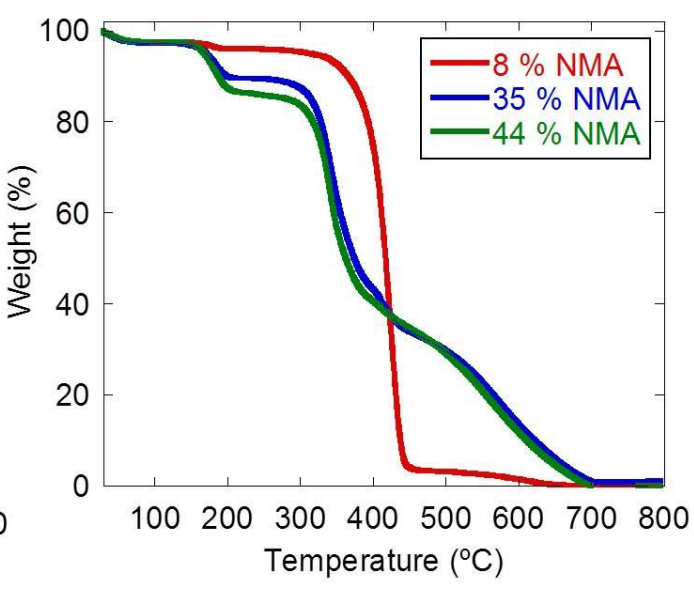

(b)

Figure 8. TGA thermographs (a) P(VCL-co-NMA) nanofiber mat containing 44 mol \% of NMA as spun and cured at $210^{\circ} \mathrm{C}$ for 1 and 7 hours, (b) as spun P(VCL-co-NMA) nanofiber mats containing different NMA mol \%.

Figure 9 compares the DSC thermograph of the as spun PN3 nanofiber mat (containing 44 mol $\%$ of NMA) as well as the mats cured at $210^{\circ} \mathrm{C}$ for $1,3,7$ and 15 hours. The sharp endothermic peak observed in the as spun sample at around $180^{\circ} \mathrm{C}$ was associated to the water and formaldehyde release due to the curing reaction. The intensity of this peak decreased as the curing time of the sample increased. Almost no peak could be observed in the sample cured for 7 hours. Interestingly, even if no differences were observed in the FTIR spectra after 30 minutes of curing at $210^{\circ} \mathrm{C}$, the DSC thermograph shows that samples cured for 1 and 3 hours still release a small amount of water and formaldehyde, suggesting that there were still unreacted NMA moieties in the sample. Although the curing process of NMA copolymers has previously been investigated by FTIR $[18,31,32]$, to our knowledge this is the first time that the curing process of the copolymers is analyzed by DSC or TGA.

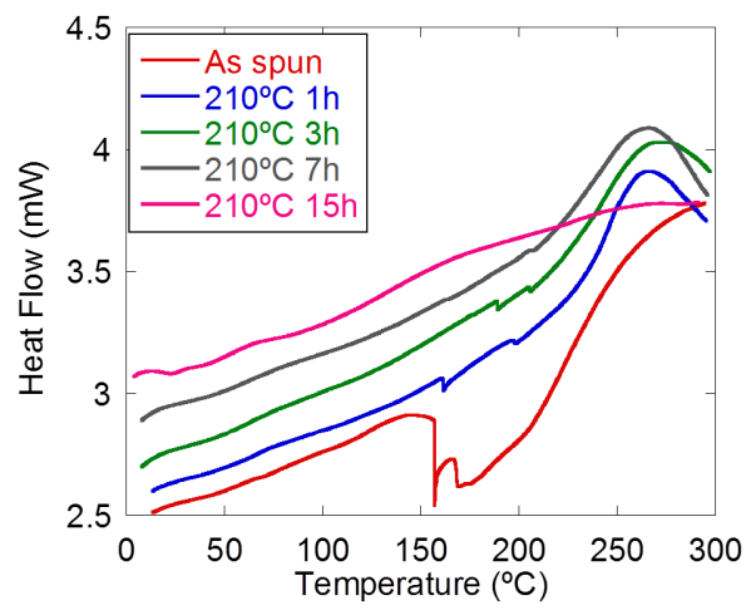

Figure 9. DSC thermographs of P(VCL-co-NMA) nanofiber mat containing 44 mol \% of NMA as spun and cured at $210^{\circ} \mathrm{C}$ for $1,3,7$ and 15 hours. 
Figure 10 (a) and (c) shows FESEM images of PN3 nanofiber mat cured at $210^{\circ} \mathrm{C}$ for 1 and 7 hours. After the curing process, samples were rinsed in water, dried at room temperature and visualized again by FESEM (Figure 10 (b) and (d)). No degradation or nanofiber breaking was observed in the cured samples. In addition fibers retained their shape after water rinsing, indicative of a successful crosslinking reaction. In the case of samples cured for just 1 hour, after water rinsing fibers looked flatter than in the case of samples cured for 7 hours.

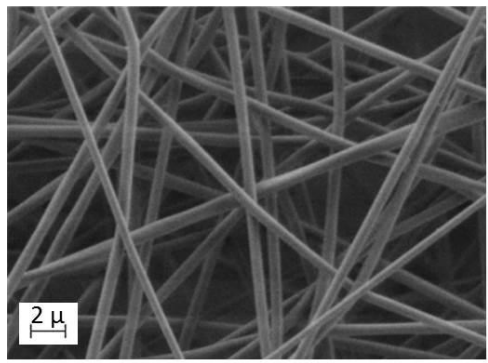

(a)

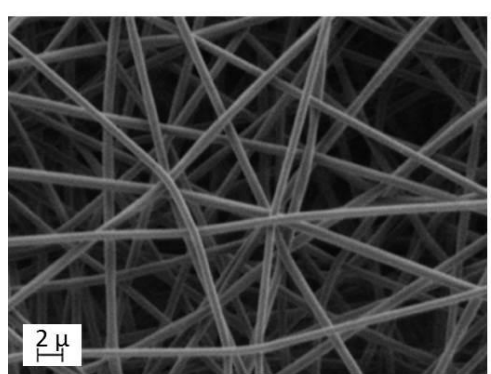

(c)

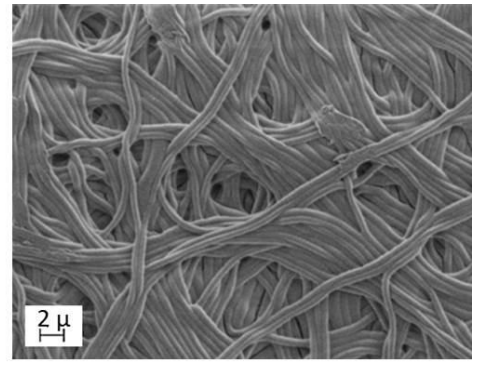

(b)

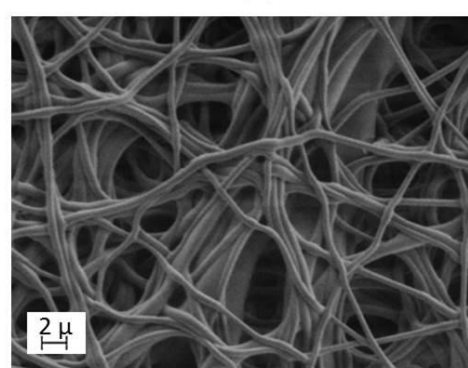

(d)

Figure 10. FESEM images of PN3 nanofiber mat; (a) cured at 210 for 1 hour, (b) cured at 210 for 1 hour after water rinsing, (c) cured at 210 for 7 hours, (d) cured at 210 for 7 hours after water rinsing.

The average fiber diameters of the cured samples ( 1 and 7 hours at $210^{\circ} \mathrm{C}$ ) are compared in Figure 11. Fiber diameters decreased after curing process, especially in the sample containing larger NMA amount. No statistical difference was observed in the sample containing $8 \mathrm{~mol} \%$ of NMA. Reduction in fiber diameter was caused by water and formaldehyde released during the crosslinking reaction.

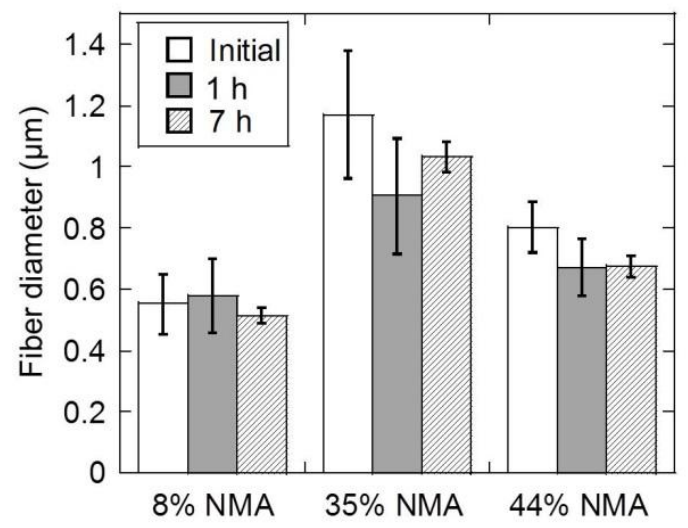


Figure 11. Average fiber diameter of P(VCL-co-NMA) nanofiber mats of different compositions after curing at $210^{\circ} \mathrm{C}$ for 1 and 7 hours.

\subsection{Temperature-responsive behavior}

The swelling-shrinking ability in water, below and above the LCST, of the cured $\left(210^{\circ} \mathrm{C}\right.$ for 1 hour) nanofiber mats was tested in order to assess their temperature-responsive behavior (Figure 12). At temperatures below the LCST, PVCL moieties of the polymer were hydrophilic leading to the nanofiber mat to swell. On the contrary, at temperatures above the LCST, the PVCL moieties become hydrophobic and collapse leading to the nanofibers to shrink. All the samples showed a good and reversible swelling-shrinking ability. Samples were immersed in water for only 5 minutes at each temperature. Thus it can be concluded that samples responded quickly to changes in temperature and their swelling ratio was recovered within each cycle.

Although no significant difference was observed between the samples containing 35 and 44 mol $\%$ of NMA, the sample containing just 8 mol $\%$ of NMA showed smaller volume changes. The reason was attributed low crosslinking density of this last sample that led to a partial dissolution of the nanofiber in water. In fact, the nanofiber containing $8 \mathrm{~mol} \%$ of NMA lost around $68 \%$ of its original weight after the experiment whereas the nanofibers containing 35 and $44 \mathrm{~mol} \%$ lost 28 and $21 \%$ respectively. A similar trend was observed by Kim et al.[32] when they study the swelling-shrinking capacity of P(NIPAm-co-NMA) nanofibers of different compositions.

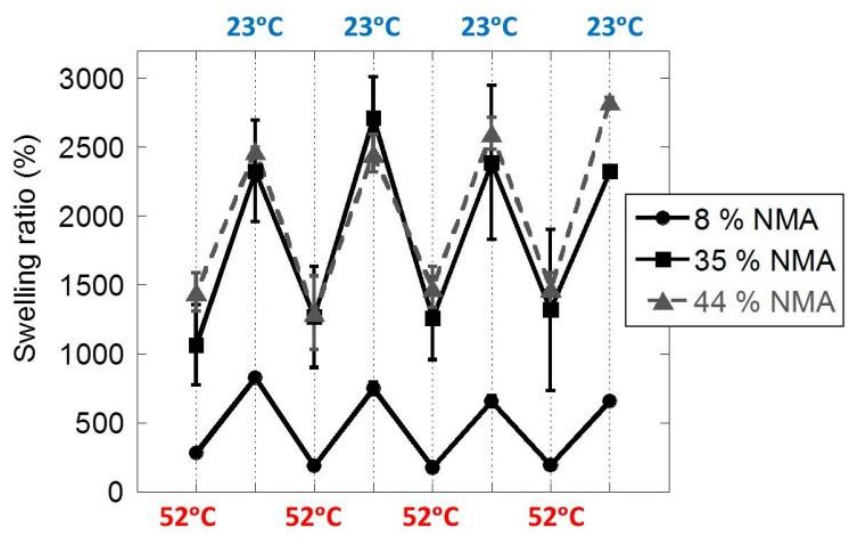

Figure 12. Temperature dependent swelling ratio of crosslinked nanofibers with different compositions. Samples were cured at $210^{\circ} \mathrm{C}$ for 1 hour.

Figure 13 compares the swelling-shrinking capacity of PN3 nanofiber mat (44 mol \% of NMA) cured at $210^{\circ} \mathrm{C}$ for 1 and 7 hours. The sample cured for 7 hours swelled less than the one cured for just one hour, suggesting that after just one hour of curing there were still unreacted NMA groups in the sample. Longer curing times led to samples with higher crosslinking density, and consequently, with lower swelling capacity. These results are in agreement with the ones observed by DSC (Figure 9) that showed that curing process was not totally completed after $1 \mathrm{~h}$ at $210^{\circ} \mathrm{C}$ even if FTIR suggested the contrary (Figure 7). 


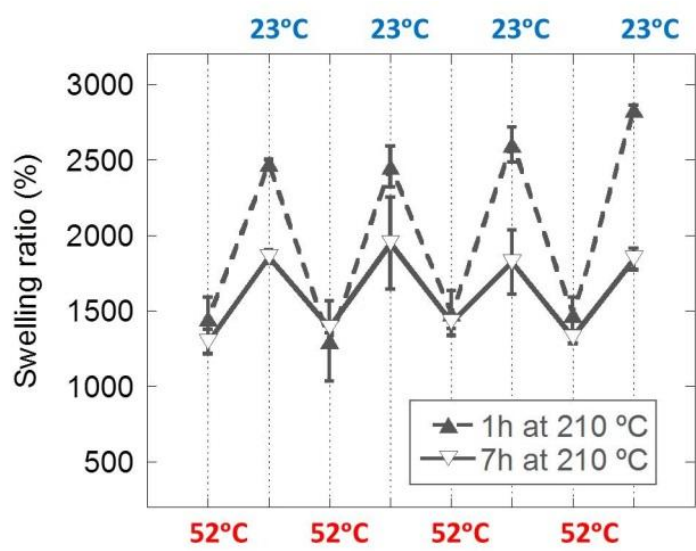

Figure 13. Swelling-shrinking ability of PN3 nanofiber mat (44 mol \% of NMA) cured at $210{ }^{\circ} \mathrm{C}$ for 1 and 7 hours.

\subsection{Temperature controlled dye release}

In order to study the controlled dye release ability of the nanofiber mats, $1 \mathrm{wt} \%$ of rhodamine B dye (as model compound) was incorporated into PN2 electrospun nanofiber mat (see section 2.6) and its release in water was studied at $23 \pm 2$ and $52 \pm 2{ }^{\circ} \mathrm{C}$. As shown in Figure 14, dye release was temperature dependent, being higher and faster at $52 \pm 2{ }^{\circ} \mathrm{C}$. The nanofiber mat exhibits higher dye release rate when the nanogel shrinks above LCST. This is an interesting result since most of the PVCL hydrogels known today are negative temperature-responsive (less release at temperature above the LCST) [6, 26]. Intriguingly, Kim and co-workers [32] also used NMA as co-monomer to create NIPAAm based hydrogel nanofibers and they also observed a positive temperature-responsive dextran release.

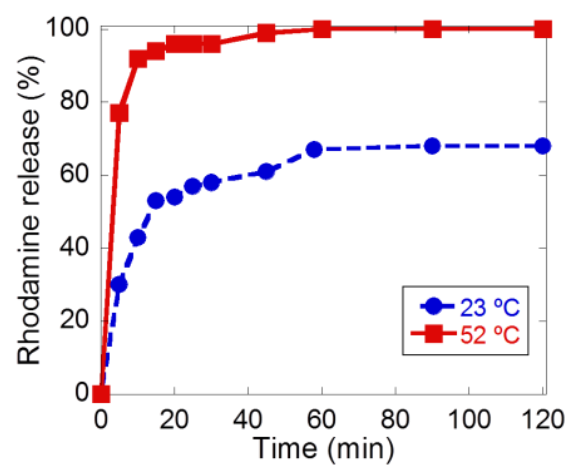

Figure 14. Release of rhodamine $B$ in water at $23 \pm 1$ and $52 \pm 2{ }^{\circ} \mathrm{C}$ for PN2 ( $35 \mathrm{~mol} \%$ of NMA) nanofiber mat.

\section{Conclusions}

In this work, $\mathrm{P}(\mathrm{VCL}-\mathrm{co}$-NMA) copolymers were successfully synthesized, characterized and electrospun to create PVCL based chemical hydrogel nanofibers for the first time. Due to the higher reactivity of NMA monomer, the amount of NMA in the final copolymer was, in all the cases, larger than in the initial reaction feed. The LCST of the copolymer increased as the NMA 
amount in the copolymer increased. Synthesized copolymers were successfully electrospun and thermally cured obtaining P(VCL-co-NMA) hydrogel nanofibers of different compositions. FTIR, DSC and TGA were used to analyze the crosslinking process. The temperature dependent responsiveness of the nanofibers was assessed by swelling-shrinking experiments below and above their LCST. All the samples responded quickly and reversibly to changes in temperature. The nanofibers containing the smallest amount of NMA (just 8 mol \% NMA), showed the smallest changes in volume as result of the partial dissolution of the sample. Longer curing times led to samples with higher crosslinking density and therefore lower swelling ability. Finally, created nanofibers also showed a temperature controlled rhodamine B dye release. The unique properties offered by these novel materials show promising applications in biosensors, controlled drug delivery and microfluidic systems.

\section{Acknowledgments}

This work was supported by the USDA National Institute of Food and Agriculture, Hatch project NYS-329402 and Hatch multistate project NC-1194 NYC-329816. This work made use of the Cornell Center for Materials Research Shared Facilities which are supported through the NSF MRSEC program (DMR-1120296). This work made use of the Nanobiotechnology Center shared research facilities at Cornell. NSF-MRI (CHE-1531632) is acknowledged for NMR instrumentation support at Cornell University,

\section{References}

[1] M.A.C. Stuart, W.T.S. Huck, J. Genzer, M. Muller, C. Ober, M. Stamm, et al., Emerging applications of stimuli-responsive polymer materials, Nat Mater. 9 (2010) 101-113.

[2] B.D. Kieviet, P.M. Schon, G.J. Vancso, Stimulus-responsive polymers and other functional polymer surfaces as components in glass microfluidic channels, Lab Chip. 14 (2014) 41594170.

[3] I. Roy, M.N. Gupta, Smart Polymeric Materials: Emerging Biochemical Applications, Chem Biol. 10 (2003) 1161-1171.

[4] Y. Qiu, K. Park, Environment-sensitive hydrogels for drug delivery, Adv Drug Deliv Rev. 53 (2001) 321-339.

[5] N.A. Cortez-Lemus, A. Licea-Claverie, Poly(N-vinylcaprolactam), a comprehensive review on a thermoresponsive polymer becoming popular, Prog Polym Sci. 53 (2016) 1-51.

[6] K. Rao, K. Rao, C.-S. Ha, Stimuli Responsive Poly(Vinyl Caprolactam) Gels for Biomedical Applications, Gels. 2 (2016) 6.

[7] H. Okuzaki, K. Kobayashi, H. Yan, Thermo-Responsive Nanofiber Mats, Macromolecules. 42 (2009) 5916-5918. 
[8] H. Chen, Y.-L. Hsieh, Ultrafine hydrogel fibers with dual temperature- and pH-responsive swelling behaviors, J Polym Sci A Polym Chem. 42 (2004) 6331-6339.

[9] X. Jin, Y.-L. Hsieh, pH-responsive swelling behavior of poly(vinyl alcohol)/poly(acrylic acid) bi-component fibrous hydrogel membranes, Polymer. 46 (2005) 5149-5160.

[10] L. Wang, P.D. Topham, O.O. Mykhaylyk, J.R. Howse, W. Bras, R.A.L. Jones, et al., Electrospinning pH-Responsive Block Copolymer Nanofibers, Adv Mater. 19 (2007) 35443548 .

[11] P. Supaphol, O. Suwantong, P. Sangsanoh, S. Srinivasan, R. Jayakumar, S.V. Nair, Electrospinning of biocompatible polymers and their potentials in biomedical applications, Adv Polym Sci. 246 (2012) 213-240.

[12] A. Greiner, J.H. Wendorff, Electrospinning: a fascinating method for the preparation of ultrathin fibers, Angew Chem Int Ed. 46 (2007) 5670-5703.

[13] A. Rogina, Electrospinning process: Versatile preparation method for biodegradable and natural polymers and biocomposite systems applied in tissue engineering and drug delivery, Appl Surf Sci. 296 (2014) 221-230.

[14] L. Persano, A. Camposeo, C. Tekmen, D. Pisignano, Industrial Upscaling of Electrospinning and Applications of Polymer Nanofibers: A Review, Macromol Mater Eng. 298 (2013) 504-520.

[15] N. Wang, Y. Zhao, L. Jiang, Low-Cost, Thermoresponsive Wettability of Surfaces: Poly(N-isopropylacrylamide)/Polystyrene Composite Films Prepared by Electrospinning, Macromol Rapid Commun. 29 (2008) 485-489.

[16] M. Chen, M. Dong, R. Havelund, V.R. Regina, R.L. Meyer, F. Besenbacher, et al., Thermo-Responsive Core-Sheath Electrospun Nanofibers from Poly (Nisopropylacrylamide)/Polycaprolactone Blends, Chem Mater. 22 (2010) 4214-4221.

[17] S.-Y. Gu, Z.-M. Wang, J.-B. Li, J. Ren, Switchable Wettability of Thermo-Responsive Biocompatible Nanofibrous Films Created by Electrospinning, Macromol Mater Eng. 295 (2010) 32-36.

[18] W.-J. Chuang, W.-Y. Chiu, Thermo-responsive nanofibers prepared from poly(Nisopropylacrylamide-co-N-methylol acrylamide), Polymer. 53 (2012) 2829-2838.

[19] A. Nykänen, S.-P. Hirvonen, H. Tenhu, R. Mezzenga, J. Ruokolainen, Wetting behaviour and direct observation of thermally responsive polystyrene-block-poly( $\mathrm{N}$-isopropylacrylamide)block-polystyrene electrospun fibres in aqueous environment, Polym Int. 63 (2014) 37-43.

[20] P. Tzeng, C.-C. Kuo, S.-T. Lin, Y.-C. Chiu, W.-C. Chen, New Thermoresponsive Luminescent Electrospun Nanofibers Prepared from Poly[2,7-(9,9-dihexylfluorene)]-blockpoly(N-isopropylacrylamide)/PMMA Blends, Macromol Chem Phys. 211 (2010) 1408-1416. 
[21] Y.-C. Chiu, Y. Chen, C.-C. Kuo, S.-H. Tung, T. Kakuchi, W.-C. Chen, Synthesis, Morphology, and Sensory Applications of Multifunctional Rod-Coil-Coil Triblock Copolymers and Their Electrospun Nanofibers, ACS Appl Mater Interfaces. 4 (2012) 3387-3395.

[22] L.-N. Chen, C.-C. Kuo, Y.-C. Chiu, W.-C. Chen, Ultra metal ions and pH sensing characteristics of thermoresponsive luminescent electrospun nanofibers prepared from poly(HPBO-co-NIPAAm-co-SA), RSC Adv. 4 (2014) 45345-45353.

[23] Y. Zhang, A.L. Yarin, Stimuli-responsive copolymers of $n$-isopropyl acrylamide with enhanced longevity in water for micro- and nanofluidics, drug delivery and non-woven applications, J Mater Chem. 19 (2009) 4732-4739.

[24] X. Lin, D. Tang, Z. Yu, Q. Feng, Stimuli-responsive electrospun nanofibers from poly(Nisopropylacrylamide)-co-poly(acrylic acid) copolymer and polyurethane, J Mater Chem B. 2 (2014) 651-658.

[25] K. Young-Jin, E. Mitsuhiro, A. Takao, Temperature-responsive electrospun nanofibers for 'on-off' switchable release of dextran, Sci Tech Adv Mater. 13 (2012) 064203.

[26] Z. Yu, H. Gu, D. Tang, H. Lv, Y. Rend, S. Gue, Fabrication of PVCL-co-PMMA nanofibers with tunable volume phase transition temperatures and maintainable shape for anticancer drug release, RSC Adv. 5 (2015) 64944-64950.

[27] P. Wilke, V. Coger, M. Nachev, S. Schachschal, N. Million, S. Barcikowski, et al., Biocompatible microgel-modified electrospun fibers for zinc ion release, Polymer. 61 (2015) 163-173.

[28] U. Paaver, I. Tamm, I. Laidmäe, A. Lust, K. Kirsimäe, P. Veski, et al., Soluplus Graft Copolymer: Potential Novel Carrier Polymer in Electrospinning of Nanofibrous Drug Delivery Systems for Wound Therapy, Biomed Res Int. 2014 (2014) 7.

[29] D. Kehren, A.C. Molano Lopez, A. Pich, Nanogel-modified polycaprolactone microfibres with controlled water uptake and degradability, Polymer. 55 (2014) 2153-2162.

[30] M. Webster, J. Miao, B. Lynch, D.S. Green, R. Jones-Sawyer, R.J. Linhardt, et al., Tunable Thermo-Responsive Poly(N-vinylcaprolactam) Cellulose Nanofibers: Synthesis, Characterization, and Fabrication, Macromol Mater Eng. 298 (2013) 447-453.

[31] L.-N. Chen, Y.-C. Chiu, J.-J. Hung, C.-C. Kuo, W.-C. Chen, Multifunctional Electrospun Nanofibers Prepared from Poly((N-isopropylacrylamide)-co-(N-hydroxymethylacrylamide)) and Their Blends with 1,2-Diaminoanthraquinone for NO Gas Detection, Macromol Chem Phys. 215 (2014) 286-294.

[32] Y.-J. Kim, M. Ebara, T. Aoyagi, Temperature-responsive electrospun nanofibers for 'onoff' switchable release of dextran, Sci Technol Adv Mater. 13 (2012) 064203-064212.

[33] S. Shah, A. Pal, R. Gude, S. Devi, Synthesis and characterization of thermo-responsive copolymeric nanoparticles of poly(methyl methacrylate-co-N-vinylcaprolactam), Eur Polym J. 46 (2010) 958-967. 
[34] I.M. Okhapkin, I.R. Nasimova, E.E. Makhaeva, A.R. Khokhlov, Effect of Complexation of Monomer Units on $\mathrm{pH}$ - and Temperature-Sensitive Properties of Poly(N-vinylcaprolactam-comethacrylic acid), Macromolecules. 36 (2003) 8130-8138.

[35] P. Supaphol, O. Suwantong, P. Sangsanoh, S. Srinivasan, R. Jayakumar, S.V. Nair. Electrospinning of Biocompatible Polymers and Their Potentials in Biomedical Applications. In: R. Jayakumar, S. Nair, editors. Biomedical Applications of Polymeric Nanofibers. Berlin, Heidelberg: Springer Berlin Heidelberg; 2012. p. 213-239.

[36] Q.P. Pham, U. Sharma, A.G. Mikos, Electrospinning of polymeric nanofibers for tissue engineering applications: a review, Tissue Eng. 12 (2006).

[37] C.S. Biswas, V.K. Patel, N.K. Vishwakarma, V.K. Tiwari, B. Maiti, P. Maiti, et al., Effects of Tacticity and Molecular Weight of Poly(N-isopropylacrylamide) on Its Glass Transition Temperature, Macromolecules. 44 (2011) 5822-5824. 
Synthesis and

Electrospining of

P(VCL-co-NMA)
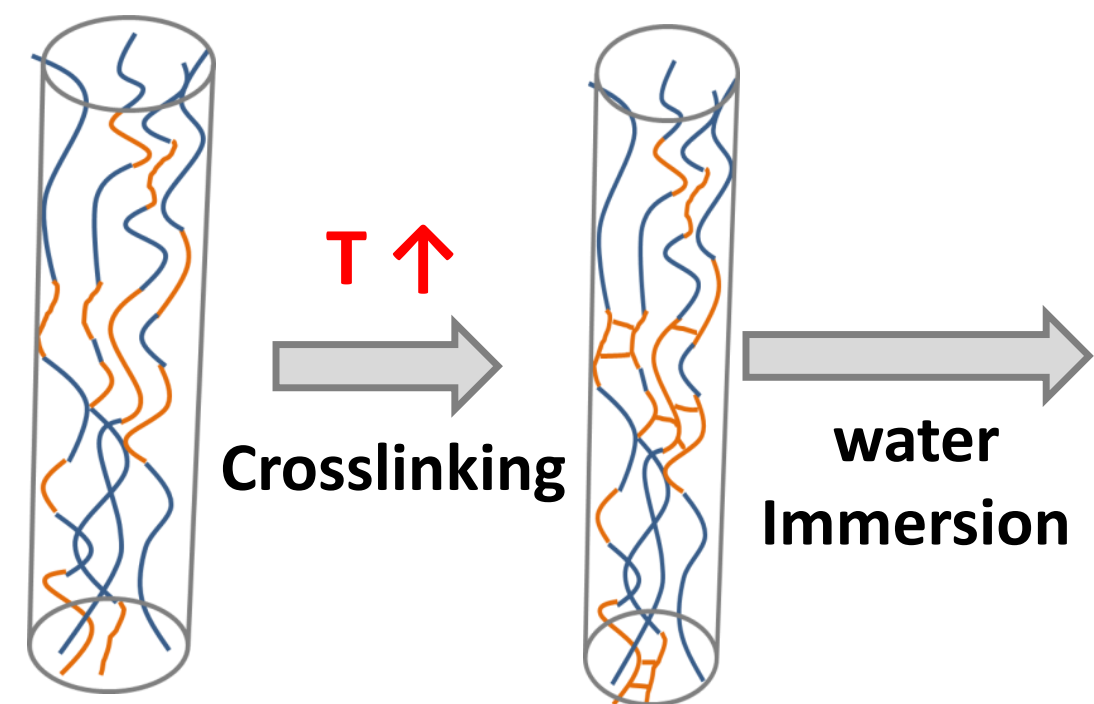

Nanofiber
Crosslinked nanofiber

\section{Temperature-responsive properties}

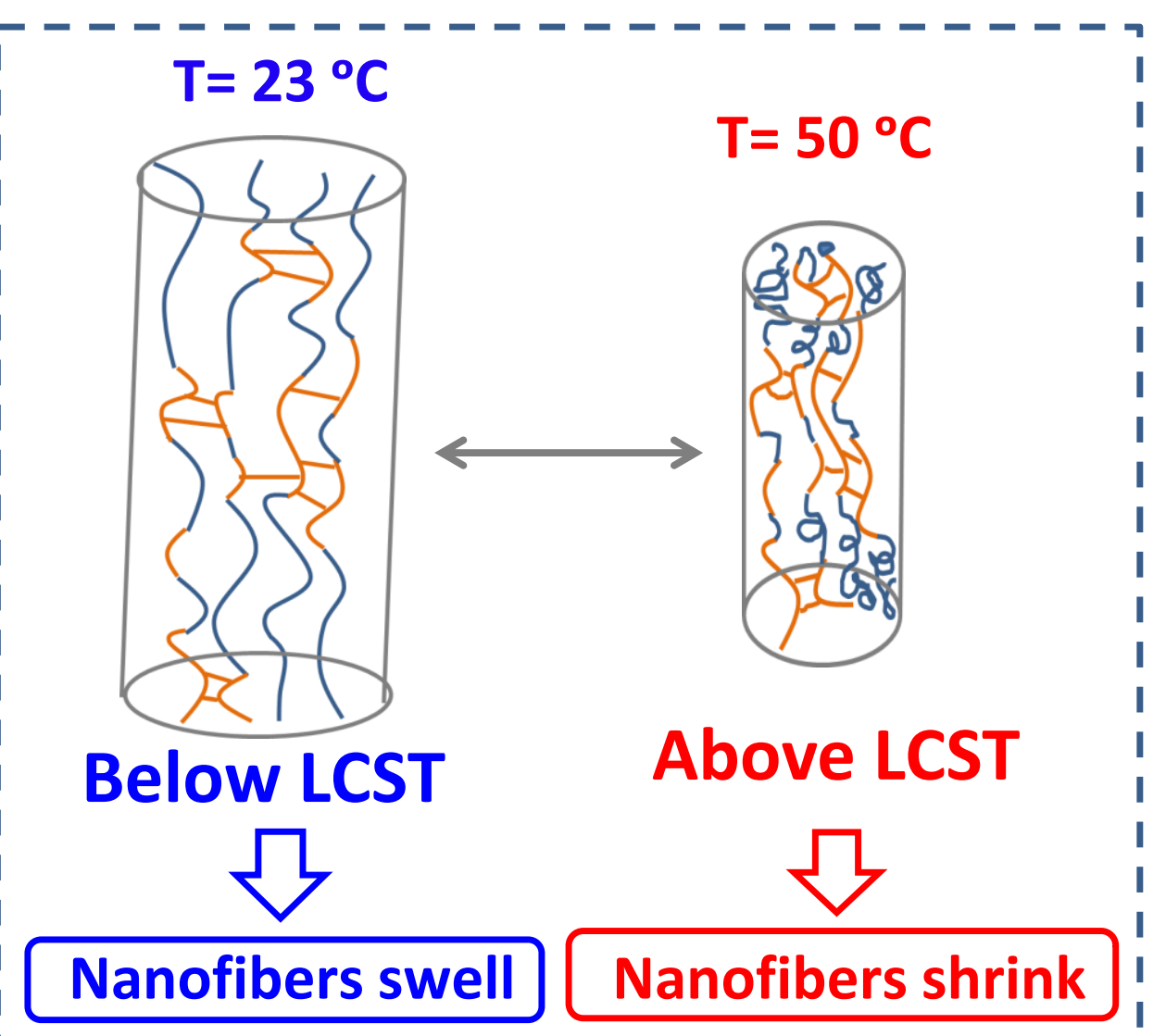

